\title{
Introducing Differential Kinematics to Mechanical Engineering Students
}

\author{
Ibrahim A. Sultan \\ School of Science and Engineering \\ University of Ballarat \\ PO Box 663, \\ Ballarat Vic 3353, Australia
}

\begin{abstract}
:
Differential kinematics offers a simplified alternative to closed form input-output equations needed to study the geometrical behaviour of linkages. For most linkages these closed form equations are either too messy or not possible to obtain. A fact that sometimes reflects negatively on how mechanical engineering students perceive the subject of Mechanism Analysis. On the other hand, differential models can easily be utilised in numerical methods designed to encourage these students to tackle even more difficult problems than currently being considered in academic programs. In this paper, an approach is presented to facilitate this process. The mathematical procedure is based on the use of matrices referred to as kinematic Jacobians. The determinants of these matrices offer invaluable insights into the linkage mobility as will be seen in the paper. These matrices are explained and used in a practice numerical example given at the end of the paper.
\end{abstract}

\section{Background:}

Mechanical engineers are often called upon to design, or maintain, machines with parts that move in fashions which are specified mathematically, i.e. linkages. That is why most mechanical engineering academic programs have in their cores a subject 
crafted to equip students with necessary knowledge in the area of mechanism analysis. This subject has traditionally been taught using graphical techniques, e.g. velocity and acceleration diagrams. As such Ceccarelli (1998) suggests, “mechanism design teaching has been strongly restricted to fundamentals and past methodologies so that generally current schemes for designing have not been and were not included in regular teaching curricula." However, with affordable personal computers becoming widely available, the use of equation-based (i.e. analytical) teaching of mechanism analysis is beginning to be more favoured by both teaching staff and students. Not only does equation-based kinematic analysis produce design-orientated insightful mathematical forms, but it also provides kinematic information that spans the whole range of the input link motion. On the other hand, graphical techniques result in information relevant only to a given input link position at which the analysis is performed. It may still be advisable, however, to briefly reflect on the velocity and acceleration diagrams just to highlight to students the vector nature of these kinematic quantities; but not to make these diagrams the core topic of the course as traditional teaching methods used to do.

Textbooks are available to introduce students to the subject of mechanism analysis using both graphical and analytical techniques. Excellent examples can be found in the works by Erdman et al. (2001), Norton (2001), Uicker et al. (2003) and Waldron and Kinzel (1999). Some of these books feature chapters in which differential models are utilised in such a fashion that for any given linkage, a Jacobian matrix is obtained and employed to calculate the velocities of various links for a given crank velocity. However, the present paper suggests that this technique should be extended, for the benefit of students, to show that more than one Jacobian matrix can be obtained for a given linkage; and these matrices can be further utilised to gain a geometric insight 
into the mobility of the linkage. The paper then proceeds to assert the notion that an input-output differential model can be conveniently utilised in a simplified numerical procedure to conduct position analysis for the mechanism.

Basically, the kinematic model is divided into three main analyses; the position analysis, the velocity analysis and eventually the acceleration analysis. If a closed form solution is possible, the position analysis should result in the following functional form;

$\mathbf{q}=\mathbf{f}(\mathbf{d}, \boldsymbol{\theta})$

where $\mathbf{d}$ is a vector representation of the constant mechanism dimensions, $\mathbf{q}=\left(q_{i}\right)$ is a vector of link freedoms (i.e. the parameters which determine the positions of various links), and the vector $\boldsymbol{\theta}=\left(\theta_{j}\right)$ signifies the freedoms of the input links. For a rotating link, the term, "freedom," is used in this paper to denote the variable angle which is measured, in a right-hand sense, from the positive X-direction to a vector attached to the link and aligned with its pivot-to-pivot direction. For a sliding link, the term refers to the distance measured from a point fixed to the line of sliding to the instantaneous position of the slider. The focus of this paper is the planar single degree of freedom linkage, which features a single entry, $\theta$, in the vector $\boldsymbol{\theta}$.

Soon after having been introduced to the basic concepts of mechanism kinematics, students quickly realise that obtaining closed form solutions as stipulated in equation (1) is almost impossible to achieve for most linkages. At this stage introducing a differential-model-based numerical technique to them would be met with appreciation and understanding. This paper is intended to encourage lecturing staff to consider 
including the topic in their undergraduate academic programs; hence, a simplified approach is adopted. However, those interested to obtain a research-level overview, on numerical techniques used for mechanism analysis, are strongly advised to consult the book by Haug (1989).

The mathematical medium which is employed to formulate the differential kinematic model is a matrix referred to here as the kinematic Jacobian. This will be introduced in the following section.

\section{Mobility and kinematic Jacobians:}

The kinematic Jacobian lies in the core of the differential approach. This is a square matrix which relates differential changes occurring to the freedoms of various links in a mechanism. Haug (1989) demonstrated techniques to obtain the kinematic Jacobian in a systematic fashion utilising the geometric properties of constraints. However, to maintain a focus on simplicity, this matrix is obtained here by differentiating the loop closure equation to develop the velocity model analytically. Textbooks which offer useful examples of these differential models are the ones by Waldron and Kinzel (1999), Uicker et al. (2003) and Erdman et al. (2001). Despite their outstanding contributions which are acknowledged by teachers and students alike, these textbooks do not exploit these differential models as much as suggested in the present paper. For closed-loop single degree of freedom linkages, these models are expressed in a form of a system of linear equations as follows;

$$
\mathbf{J}_{\theta}\left(\mathbf{d}_{q}, \mathbf{q}\right) \dot{\mathbf{q}}=\mathbf{g}_{\theta}\left(\mathbf{d}_{\theta}, \theta\right) \dot{\theta}
$$

where $\theta$ is the freedom of any link not represented in the vector $\mathbf{q}, \mathbf{d}_{q}$ and $\mathbf{d}_{\theta}$ are vectors of constant mechanism dimensions, and the dots signify differentiation with 
respect to time. In the above equation, $\mathbf{g}_{\theta}\left(\mathbf{d}_{\theta}, \theta\right)$ is the right-hand side vector of the linear system, and $\mathbf{J}_{\theta}\left(\mathbf{d}_{q}, \mathbf{q}\right)$ is an $n \times n$ kinematic Jacobian matrix of the linkage, where $n$ is the number of unknown link-velocities. Mobility analysis can be based on the mathematical characteristics of the linear set given in (2), in addition to the geometric aspects of rigid linkages. Of particular importance to the analysis is the case in which the determinant, $\left|\mathbf{J}_{\theta}\left(\mathbf{d}_{q}, \mathbf{q}\right)\right|$, of the Jacobian $\mathbf{J}_{\theta}\left(\mathbf{d}_{q}, \mathbf{q}\right)$ vanishes. In this singular case, it is advisable to re-arrange equation (2) in such a fashion that the velocity, say $\dot{\beta}$, of a different link replaces $\dot{\theta}$ on the right-hand side of the equation. The corresponding determinant, $\left|\mathbf{J}_{\beta}\left(\mathbf{d}_{\text {qnew }}, \mathbf{q}_{\text {new }}\right)\right|$, of the new resulting Jacobian should also be obtained and used to distinguish amongst the following cases;

Case 1: if $\left|\mathbf{J}_{\theta}\left(\mathbf{d}_{q}, \mathbf{q}\right)\right|=\left|\mathbf{J}_{\beta}\left(\mathbf{d}_{\text {qnew }}, \mathbf{q}_{\text {new }}\right)\right|=0$, the mechanism becomes singular in such a way that geometric constraints are lost. Some examples on the occurrence of this situation are listed as follows;

i. A slider-crank mechanism where the crank length is set equal to the length of the connecting rod. The crank will become aligned in the vertical position with the connecting rod to suggest that the slider can, geometrically, remain stationary whilst the crank continues its rotational motion.

ii. A parallelogram four-bar chain at the position in which all links are simultaneously aligned with the ground link.

iii. A quick-return linkage with the crank length set equal to the distance between the rocker stationary pivot and the crank 
stationary pivot. Such an arrangement is singular at the instant when the crank end passes by the rocker stationary pivot.

Case 2: if $\left|\mathbf{J}_{\theta}\left(\mathbf{d}_{q}, \mathbf{q}\right)\right|=0$ and $\left|\mathbf{J}_{\beta}\left(\mathbf{d}_{\text {qnew }}, \mathbf{q}_{\text {new }}\right)\right| \neq 0$ then $\dot{\theta}=0$; if $\dot{\theta}$ is the velocity of the input link, this suggests a lock-up of the mechanism.

Case 3: if $\left|\mathbf{J}_{\theta}\left(\mathbf{d}_{q}, \mathbf{q}\right)\right|=0$ and $\left|\mathbf{J}_{\beta}\left(\mathbf{d}_{\text {qnew }}, \mathbf{q}_{\text {new }}\right)\right| \neq 0$ then $\dot{\theta}=0$; if $\dot{\theta}$ is the velocity of any link other than the input link, then either this link is at the end of a stroke, or going through a dwell. This condition could be employed to find the stationary positions of various links.

The next section offers an example on how to use the Jacobians to gain an insight into the kinematic behaviour of a four-bar linkage.

\section{The Jacobians of the four-bar chain:}

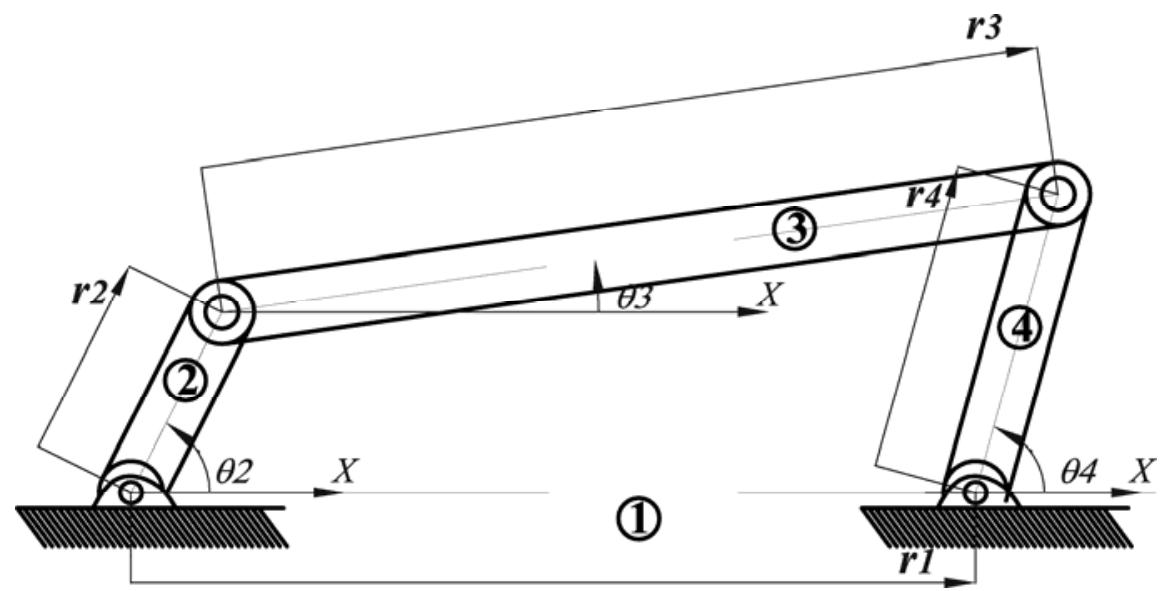

Figure 1. A four-bar chain

A four-bar chain is shown in Figure (1). As measured from the positive X-direction, the freedoms of various links $\left(0, \theta_{2}, \theta_{3}, \theta_{4}\right)$, and their lengths, $\left(r_{1}, r_{2}, r_{3}, r_{4}\right)$, are used to write the position vectors $\mathbf{r}_{1}=r_{1} e^{j 0}, \mathbf{r}_{2}=r_{2} e^{j \theta_{2}}, \mathbf{r}_{3}=r_{3} e^{j \theta_{3}}$ and $\mathbf{r}_{4}=r_{4} e^{j \theta_{4}}$ where $j$ is the complex number operator and the dimensions are pointed out on the drawing. The 
loop closure equation can then be written $\left(\mathbf{r}_{2}+\mathbf{r}_{3}=\mathbf{r}_{1}+\mathbf{r}_{4}\right)$ and differentiated to obtain the following expression;

$$
r_{2} \dot{\theta}_{2} e^{j\left(\theta_{2}+\pi / 2\right)}+r_{3} \dot{\theta}_{3} e^{j\left(\theta_{3}+\pi / 2\right)}-r_{4} \dot{\theta}_{4} e^{j\left(\theta_{4}+\pi / 2\right)}=\mathbf{0}
$$

which is then resolved in the $\mathrm{X}$ - and Y-directions as follows;

$-r_{2} \sin \theta_{2} \dot{\theta}_{2}-r_{3} \sin \theta_{3} \dot{\theta}_{3}+r_{4} \sin \theta_{4} \dot{\theta}_{4}=0$

and

$r_{2} \cos \theta_{2} \dot{\theta}_{2}+r_{3} \cos \theta_{3} \dot{\theta}_{3}-r_{4} \cos \theta_{4} \dot{\theta}_{4}=0$

Equations (4) can be re-arranged such that 3 kinematic expressions are written as follows;

$$
\left[\mathbf{J}_{\theta_{2}}\right]\left[\begin{array}{c}
\dot{\theta}_{3} \\
\dot{\theta}_{4}
\end{array}\right]=\left[\begin{array}{c}
r_{2} \sin \theta_{2} \\
-r_{2} \cos \theta_{2}
\end{array}\right]^{\dot{\theta}_{2}},\left[\mathbf{J}_{\theta_{3}}\right]\left[\begin{array}{l}
\dot{\theta}_{2} \\
\dot{\theta}_{4}
\end{array}\right]=\left[\begin{array}{c}
r_{3} \sin \theta_{3} \\
-r_{3} \cos \theta_{3}
\end{array}\right]^{\dot{\theta}_{3}} \text { and }\left[\mathbf{J}_{\theta_{4}}\right]\left[\begin{array}{l}
\dot{\theta}_{2} \\
\dot{\theta}_{3}
\end{array}\right]=\left[\begin{array}{c}
-r_{4} \sin \theta_{4} \\
r_{4} \cos \theta_{4}
\end{array}\right] \dot{\theta}_{4}
$$

where the Jacobian matrices are given as follows;

$$
\begin{aligned}
\mathbf{J}_{\theta_{2}} & =\left[\begin{array}{cc}
-r_{3} \sin \theta_{3} & r_{4} \sin \theta_{4} \\
r_{3} \cos \theta_{3} & -r_{4} \cos \theta_{4}
\end{array}\right], \\
\mathbf{J}_{\theta_{3}} & =\left[\begin{array}{cc}
-r_{2} \sin \theta_{2} & r_{4} \sin \theta_{4} \\
r_{2} \cos \theta_{2} & -r_{4} \cos \theta_{4}
\end{array}\right] \text { and } \\
\mathbf{J}_{\theta_{4}} & =\left[\begin{array}{cc}
-r_{2} \sin \theta_{2} & -r_{3} \sin \theta_{3} \\
r_{2} \cos \theta_{2} & r_{3} \cos \theta_{3}
\end{array}\right]
\end{aligned}
$$

The determinants of these matrices can now be expressed as follows; $\left|\mathbf{J}_{\theta_{2}}\right|=r_{3} r_{4} \sin \left(\theta_{3}-\theta_{4}\right),\left|\mathbf{J}_{\theta_{3}}\right|=r_{2} r_{4} \sin \left(\theta_{2}-\theta_{4}\right)$ and $\left|\mathbf{J}_{\theta_{4}}\right|=r_{3} r_{2} \sin \left(\theta_{3}-\theta_{2}\right)$

Setting $\left|\mathbf{J}_{\theta_{2}}\right|=0$ implies the condition $\theta_{3}=\theta_{4}\left(\right.$ or $\left.\theta_{4}+\pi\right)$; i.e. links number 3 and 4 are aligned. Obviously for a general case, where the lengths are selected such that 
$\left|\mathbf{J}_{\theta_{3}}\right| \neq 0$ or $\left|\mathbf{J}_{\theta_{4}}\right| \neq 0$, the condition suggests a lock-up. As indicated above, if $\left|\mathbf{J}_{\theta_{2}}\right|=0$, proving that either $\left|\mathbf{J}_{\theta_{3}}\right| \neq 0$ or $\left|\mathbf{J}_{\theta_{4}}\right| \neq 0$ is sufficient to conclude the lock-up.

Likewise, setting $\left|\mathbf{J}_{\theta_{4}}\right|=0$ implies the condition $\theta_{3}=\theta_{2}\left(\right.$ or $\left.\theta_{2}+\pi\right)$ which, if supported by either $\left|\mathbf{J}_{\theta_{2}}\right| \neq 0$ or $\left|\mathbf{J}_{\theta_{3}}\right| \neq 0$, suggests that link number 4 is at its extreme positions. Similar analysis can readily be carried out to discover that for link 3 to arrive at its extreme positions, the crank and the rockers have to be parallel to each other.

If the mechanism was designed in such a way that $r_{1}=r_{3}$ and $r_{2}=r_{4}$ (i.e. a parallelogram where $\theta_{2}=\theta_{4}$ and $\theta_{3}=0$ during the mechanism motion), $\left|\mathbf{J}_{\theta_{3}}\right|$ will always be equal to 0 , even when the other two determinants have nonzero values. This reflects the fact that $\dot{\theta}_{3}$ is always 0 , and the coupler is always parallel to the ground link. At the two positions, $\theta_{2}=\theta_{4}=0$ and $\theta_{2}=\theta_{4}=\pi$, where all links are aligned with the ground, the three determinants will simultaneously vanish to highlight the occurrence of singularity. The reader is encouraged to repeat the mobility analysis presented in this section on a slider-crank linkage, and on a quickreturn linkage.

\section{Computational kinematics:}

After having been introduced to the differential model in equation (2) and to the level of insight which is obtainable from the Jacobian matrices, students should now be ready for the concepts of computational kinematics. The initial step here is to arrange equation (2) in such a way that $\dot{\theta}$ is the velocity of the input. An approximation is then adopted where for a small time interval, $\delta t$, various time-derivatives may be 
replaced by ratios of infinitesimal changes such that $\frac{d \mathbf{q}}{d t} \approx \frac{\delta \mathbf{q}}{\delta t}$ and $\frac{d \theta}{d t} \approx \frac{\delta \theta}{\delta t}$. This leads to a form of equation (2) where $\delta t$ is cancelled out on both sides, and the small differential changes, $\delta \mathbf{q}$, which occur as a result of a small differential motion, $\delta \theta$, can be calculated as follows;

$\delta \mathbf{q}_{i}=\mathbf{J}_{\theta}\left(\mathbf{d}_{q}, \mathbf{q}_{i}\right)^{-1} \mathbf{g}_{\theta}\left(\mathbf{d}_{\theta}, \theta_{i}\right) \delta \theta$

where $i=0,1,2, \cdots, N+1$ is the step number, and the updated values of the link freedoms are expressed as follows;

$$
\left.\begin{array}{l}
\mathbf{q}_{i+1}=\mathbf{q}_{i}+\delta \mathbf{q}_{i} \\
\theta_{i+1}=\theta_{i}+\delta \theta
\end{array}\right\}
$$

Also the velocities and accelerations of various links can be calculated with a reasonable degree of accuracy as follows;

$$
\left.\begin{array}{rl}
\dot{\mathbf{q}}_{i} & =\frac{\delta \mathbf{q}_{i}}{\delta t} \\
\ddot{\mathbf{q}}_{i} & =\frac{\delta \mathbf{q}_{i+1}-\delta \mathbf{q}_{i}}{\delta t^{2}}
\end{array}\right\}
$$

The expression given for the acceleration in equation (9) reveals why the number of intervals used for the calculations, $N+1$, should be greater than the number of intervals, $N$, on the $\theta$-axis by 1 .

The technique presented above employs the well known Euler method to find a numerical solution to the set of simultaneous differential equations represented by the mechanism differential model. A differential-model-based technique is ideal to introduce to undergraduate students as a numerical tool for computational kinematics due to its non-iterative nature, ease of programming and ability to calculate the velocity and acceleration of links as easily as offered by equation (9). That is why a differential-model-based technique has been adopted in this paper rather than a root- 
finding technique, such as the Newton-Raphson method. Being iterative in nature, root finding techniques are computationally costly, and more suitable for finding solutions only for a few data points widely separated on the $\theta$-axis. This implies that calculations can be performed at a much smaller number of data points than needed by the Euler method. However, a too small number of data points will impair the resolution of the obtained results and important information on the kinematic behaviour of the linkage may be missed. Moreover, after the roots have been iteratively found at every data point, the velocity and acceleration solutions still have to be calculated by solving the two linear systems obtained by taking the first and second time-derivatives of the loop closure equations.

Despite its simplicity, the Euler method is capable of producing accurate outcome so long as the number of intervals selected on the $\theta$-axis is large enough as will be shown by the practice example given in the next section. The example also features a function proposed to quantify the calculation error, and monitor its development during the course of computation.

\section{Practice example:}

For this example, it is required to produce graphs to describe the kinematic behaviour of the linkage sketched in Figure (2); given a constant input velocity, $\dot{\theta}_{2}$, for link 2. After a few attempts to obtain a closed form solution to relate various link freedoms to the freedom of link 2 (i.e. $\theta_{2}$ ), students usually realise the degree of difficulty featured in this problem and start looking for alternative techniques. At this stage, they are encouraged to consider the use of a differential-model-based numerical method as described above. The analysis sets out by writing the loop closure equations as follows; 


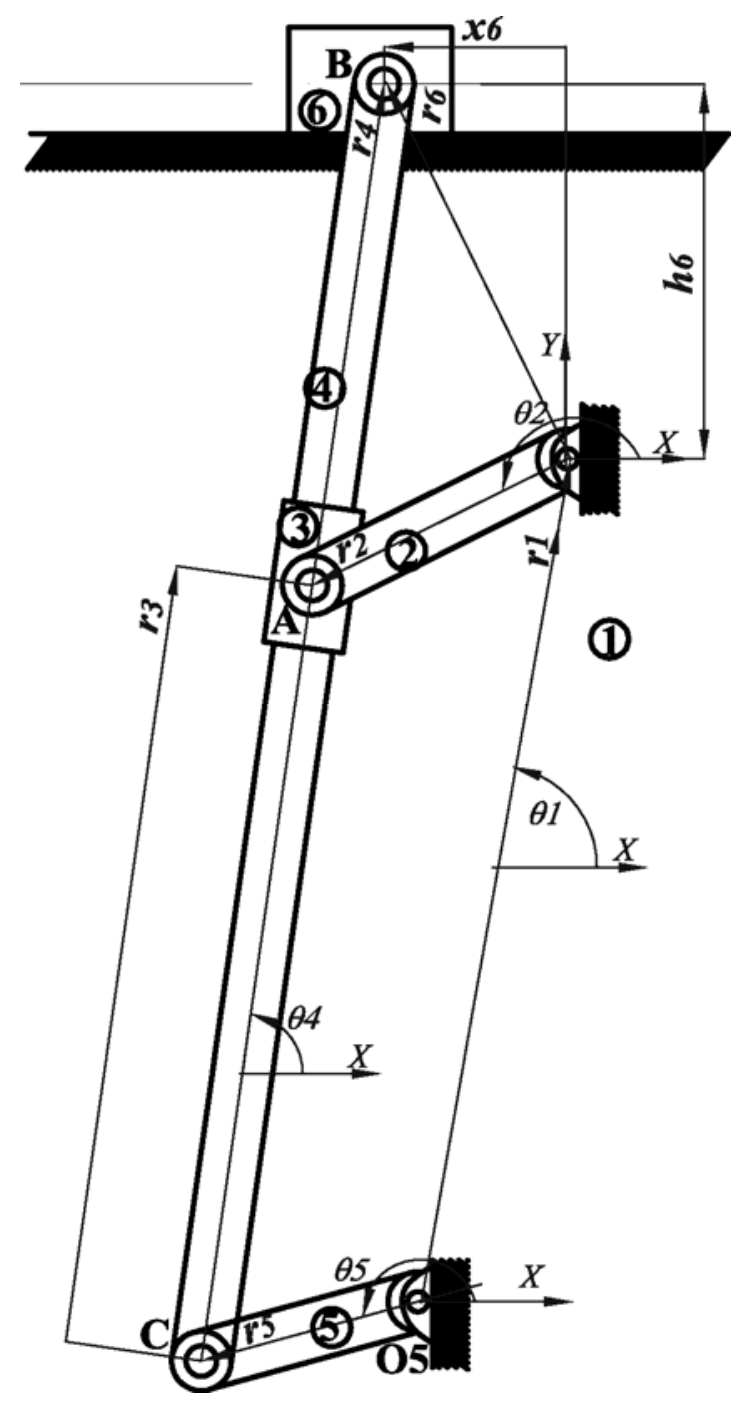

Figure 2. A linkage used for the practice example - not to scale.

$\left.\begin{array}{l}\mathbf{r}_{5}+\mathbf{r}_{3}=\mathbf{r}_{1}+\mathbf{r}_{2} \\ \mathbf{r}_{5}+\mathbf{r}_{4}=\mathbf{r}_{1}+\mathbf{r}_{6}\end{array}\right\}$

where $\mathbf{r}_{1}=r_{1} e^{j \theta_{1}}, \mathbf{r}_{2}=r_{2} e^{j \theta_{2}}, \mathbf{r}_{3}=r_{3} e^{j \theta_{4}}, \mathbf{r}_{4}=r_{4} e^{j \theta_{4}}, \mathbf{r}_{5}=r_{5} e^{j \theta_{5}}$ and $\mathbf{r}_{6}=x_{6} e^{j 0}+h_{6} e^{j \pi / 2}$.

The various dimensions are described on the drawing, and the differentiation process will result in the following expression for the computational model; 
$\left[\mathbf{J}_{\theta_{2}}\right]\left[\begin{array}{l}\delta \theta_{5} \\ \delta \theta_{4} \\ \delta r_{3} \\ \delta x_{6}\end{array}\right]=\left[\begin{array}{c}-r_{2} \sin \theta_{2} \\ r_{2} \cos \theta_{2} \\ 0 \\ 0\end{array}\right] \delta \theta_{2}$

where the Jacobian, $\mathbf{J}_{\theta_{2}}$, is given as follows;

$$
\mathbf{J}_{\theta_{2}}=\left[\begin{array}{cccc}
-r_{5} \sin \theta_{5} & -r_{3} \sin \theta_{4} & \cos \theta_{4} & 0 \\
r_{5} \cos \theta_{5} & r_{3} \cos \theta_{4} & \sin \theta_{4} & 0 \\
-r_{5} \sin \theta_{5} & -r_{4} \sin \theta_{4} & 0 & -1 \\
r_{5} \cos \theta_{5} & r_{4} \cos \theta_{4} & 0 & 0
\end{array}\right]
$$

It would be advisable to obtain another kinematic Jacobian, say $\mathbf{J}_{\theta_{4}}$. Unless otherwise required, the determinant of this second Jacobian would be calculated only if it was discovered that $\left|\mathbf{J}_{\theta_{2}}\right|$ vanishes during the course of computation. In this case, if $\left|\mathbf{J}_{\theta_{4}}\right|$ also vanishes at the same value of $\theta_{2}$, the linkage would be singular, which may warrant re-synthesis of its dimensions. On the other hand, if $\left|\mathbf{J}_{\theta_{4}}\right|$ does not vanish at the value of $\theta_{2}$ where $\left|\mathbf{J}_{\theta_{2}}\right|$ vanishes, a lock-up would be concluded which is also a problem that warrants re-synthesis. In fact, for the mechanism to function properly, the value of $\left|\mathbf{J}_{\theta_{2}}\right|$ should always be nonzero. In other words, this value should remain either positive or negative during the course of computations, and should never intersect the $\theta_{2}$-axis, as shown in Figure (7). This remark does not apply to the determinants of other kinematic Jacobians present in the mechanism, i. e. $\left|\mathbf{J}_{\theta_{4}}\right|,\left|\mathbf{J}_{r_{3}}\right|,\left|\mathbf{J}_{\theta_{5}}\right|$ nor $\left|\mathbf{J}_{x_{6}}\right|$, due to the fact that these determinants vanish at the extreme positions of their corresponding links. They will also change sign as their corresponding links change direction of motion. 
The loop closure equation which has the position vector of the input link can conveniently be used to quantify the accuracy of calculations if arranged in the following form;

$$
\left(\mathbf{r}_{5}+\mathbf{r}_{3}-\mathbf{r}_{1}\right)-\mathbf{r}_{2}=\mathbf{0}
$$

At a given angle $\theta_{2(i+1)}$, which is the value of $\theta_{2}$ as updated at the end of step number $i$, the second vector term of equation (13), $\mathbf{r}_{2}$, is accurately defined. Had the vector sum $\left(\mathbf{r}_{5}+\mathbf{r}_{3}-\mathbf{r}_{1}\right)$ also been accurately defined, it would have been exactly equal to $\mathbf{r}_{2}$ so that the dot-product of the two vectors should produce the exact same scalar value of $r_{2}^{2}$. Subsequently, a convenient approach to quantify the error is to subtract this dot-product from $r_{2}^{2}$ and then divide the difference by $r_{2}^{2}$ to obtain a relative perspective for this error. Mathematically, this can be expressed as follows;

$e r_{i+1}=\left(1-\frac{\left(r_{5} e^{j \theta_{5(i+1)}}+r_{3(i+1)} e^{j \theta_{4(i+1)}}-r_{1} e^{j \theta_{1}}\right) \bullet r_{2} e^{j \theta_{2(i+1)}}}{r_{2}^{2}}\right) \times 100$

where $e r_{i+1}$ is the percentage error as calculated at the end of step number $i$.

The numerical value of the error function for the practice example is depicted in Figure (8). The greatest deviation was found to fall below $-0.07 \%$, which reveals the high level of accuracy obtainable from such a simplified computational approach.

The above procedure has been coded in a MathCAD function to describe the computational model of the example problem. The constant dimensions used for the simulation are as follows; $\theta_{1}=76^{\circ}, r_{1}=42 \mathrm{~cm}, r_{2}=13.027 \mathrm{~cm}, r_{4}=63.6 \mathrm{~cm}$ and $r_{5}=15 \mathrm{~cm}$. The initial congruent values of various freedoms have been measured, of a CAD drawing, as follows; $\theta_{2}=0, \theta_{4}=61^{\circ}, \theta_{5}=59^{\circ}, \quad x_{6}=28.399 \mathrm{~cm}$ and $r_{3}=31.893 \mathrm{~cm}$. It was also found from the CAD drawing that $h_{6}=27.73 \mathrm{~cm}$, even 
though it is not needed for the differential analysis. The number of steps, $N$, is assigned the value of 7200, which implies that $\delta \theta_{2}=\frac{2 \pi}{7200} \mathrm{rad}$. For the velocity and acceleration calculations, the constant speed, $\dot{\theta}_{2}$, of the crank is given as $100 \mathrm{rpm}$, which implies that $\delta t=\frac{60}{7200 \times 100} \mathrm{sec}$. The outcome of the analysis is shown in Figures 3 to 7 . In these figures, the values given for various kinematic variables (position, velocity, acceleration and Jacobian determinant) are expressed in relative terms as follows;

$$
\mathbf{v}_{i}^{\text {relative }}=\frac{\mathbf{v}_{i}}{v_{\max }}
$$

where $\mathbf{v}_{i}$ is a vector that contains the values for any kinematic variable. Also, $v_{\max }$ signifies the maximum absolute value inside $\mathbf{v}_{i}$ and $\mathbf{v}_{i}^{\text {relative }}$ is the relative form of the vector.

A closed-form solution for the practice example is presented in the attached appendix. Table 1 below features the values of the mechanism freedoms as obtained using both the closed-form solution and the simplified numerical method shown above. To highlight the accuracy level obtainable using the computational technique, the analysis was conducted at four broadly-spaced values for $\theta_{2}$ as shown in the table. 


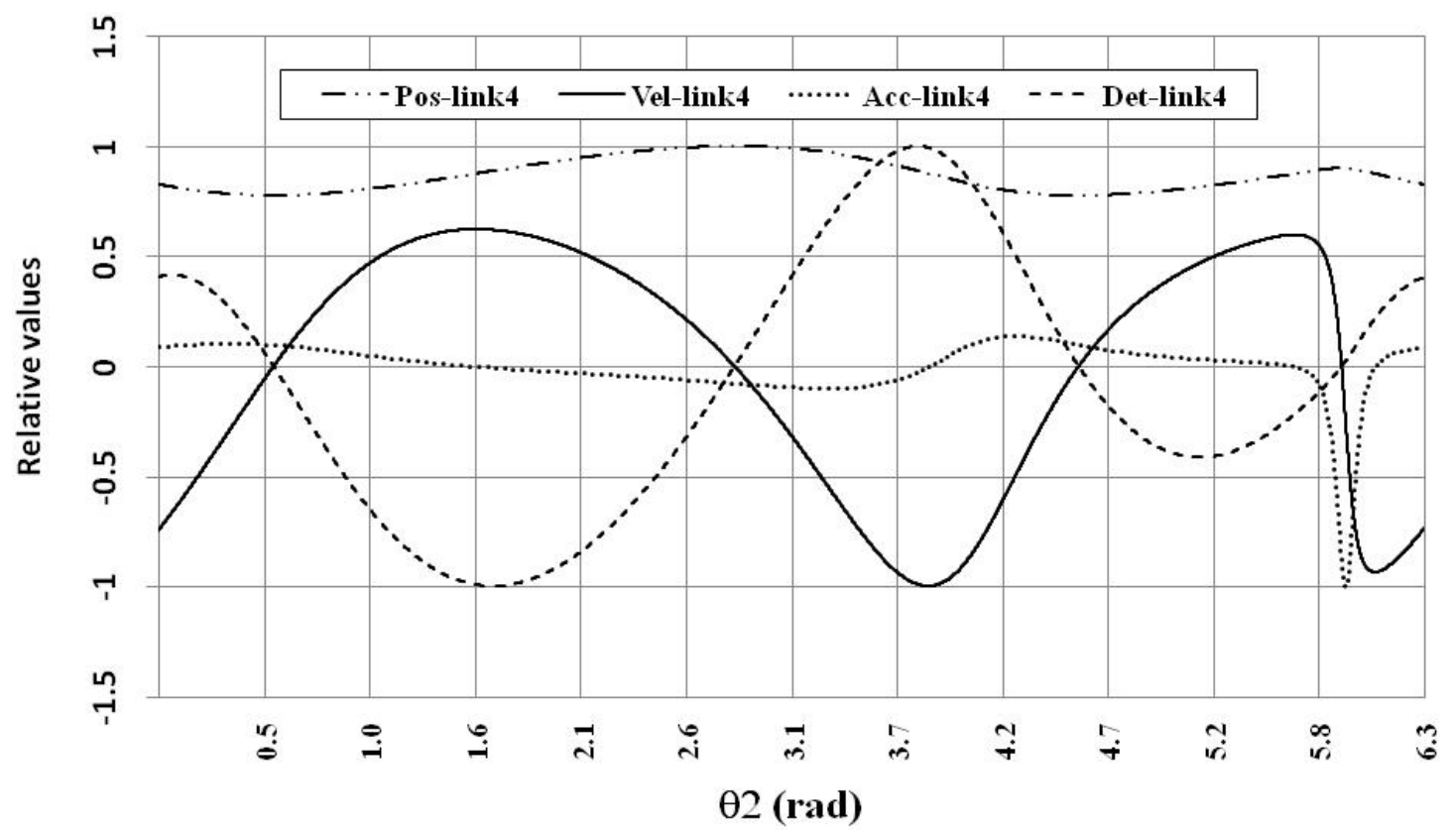

Figure 3. Position, velocity, acceleration and Jacobian determinant of link 4.

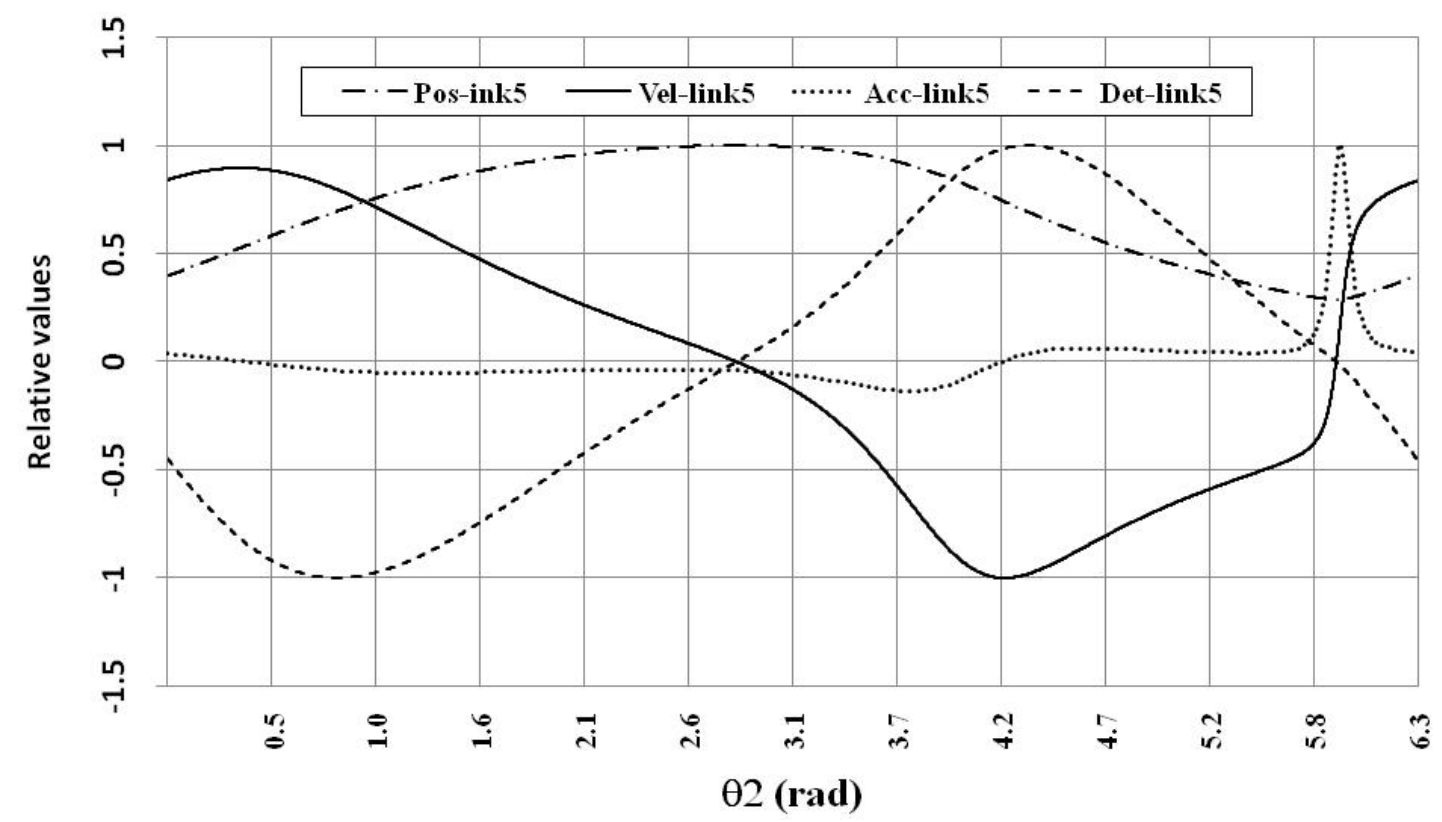

Figure 4. Position, velocity, acceleration and Jacobian determinant of link 5. 


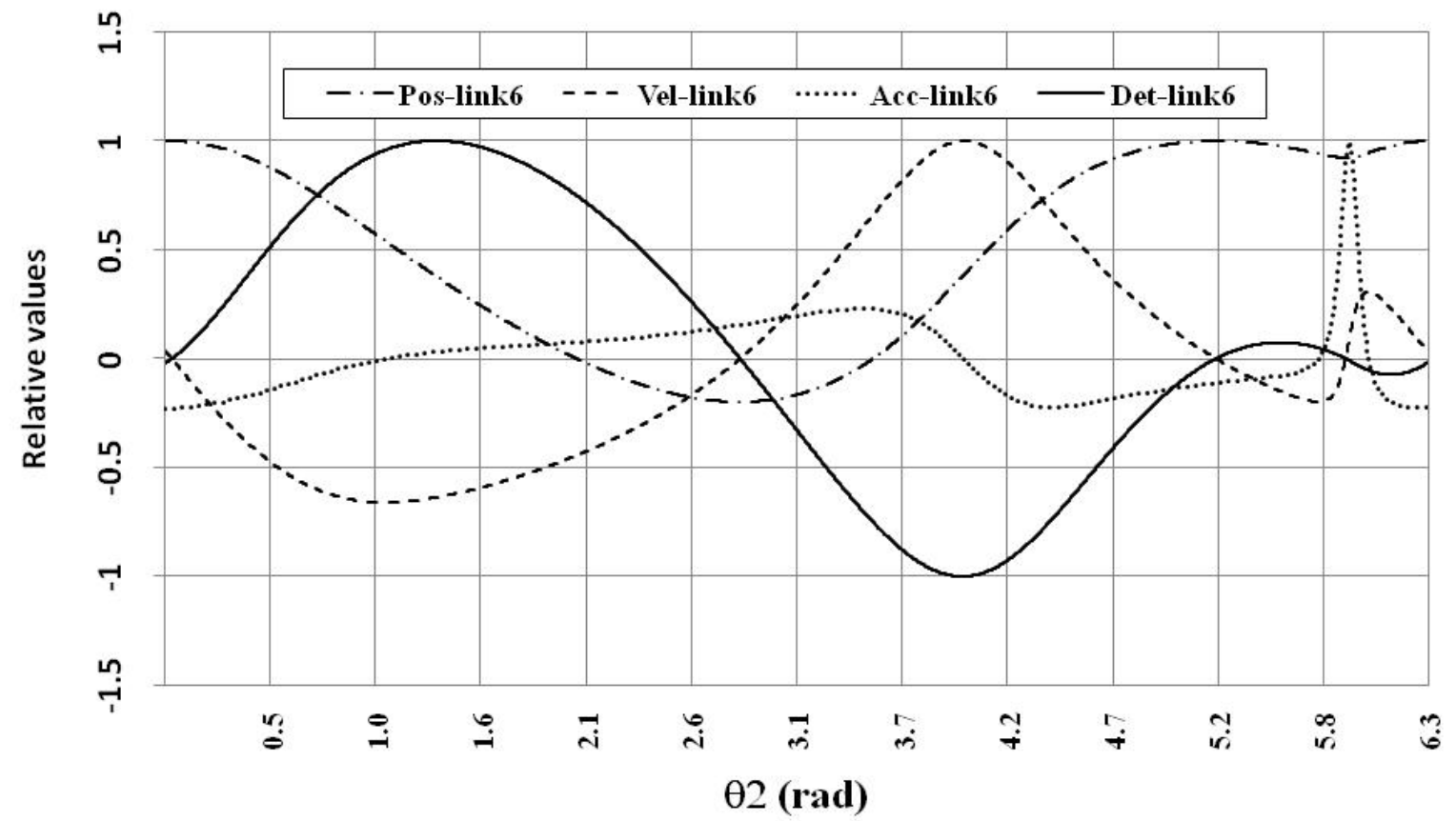

Figure 5. Position, velocity, acceleration and Jacobian determinant of slider 6

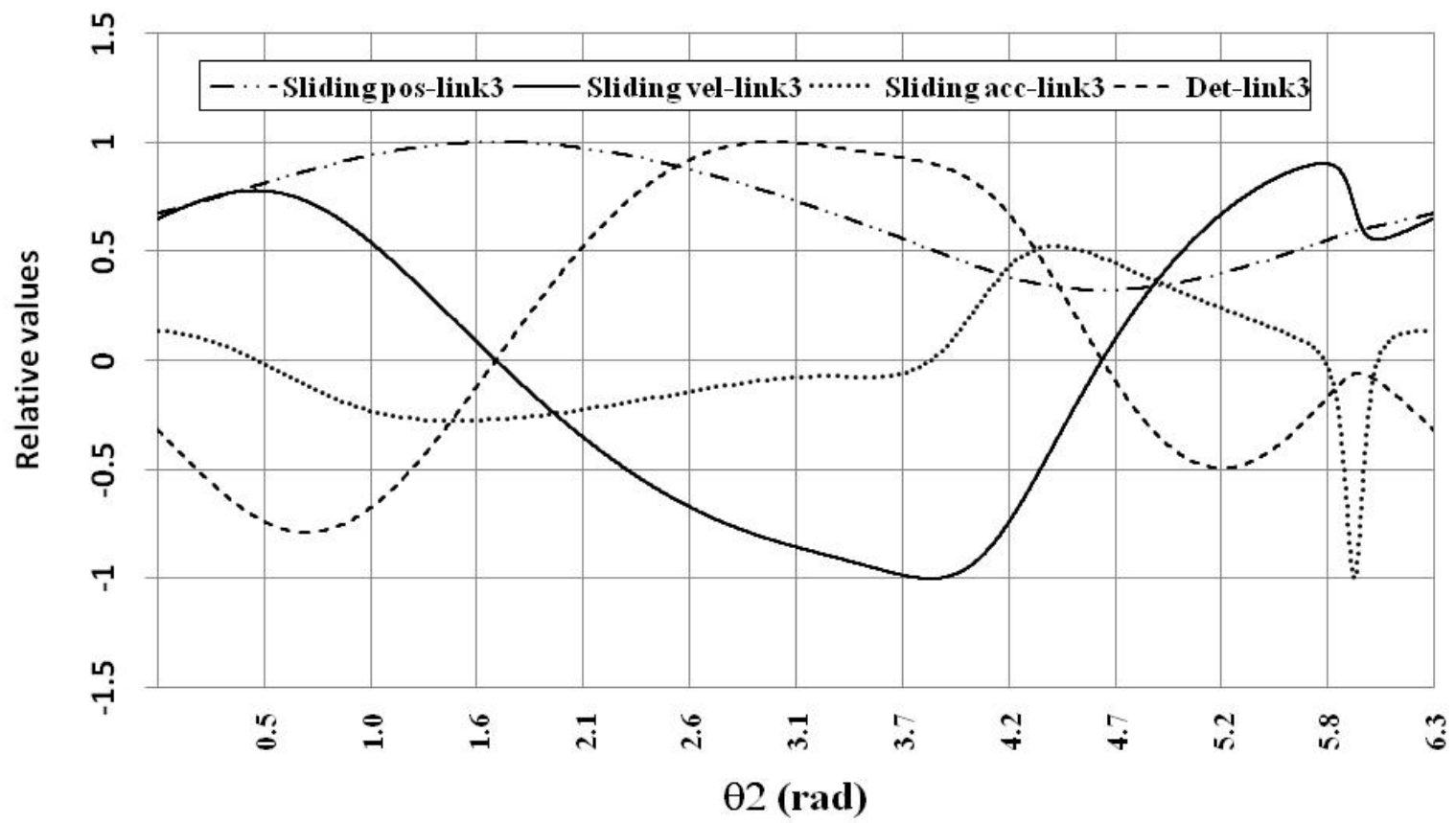

Figure 6. Axial position, velocity, acceleration and Jacobian determinant of slider 3 


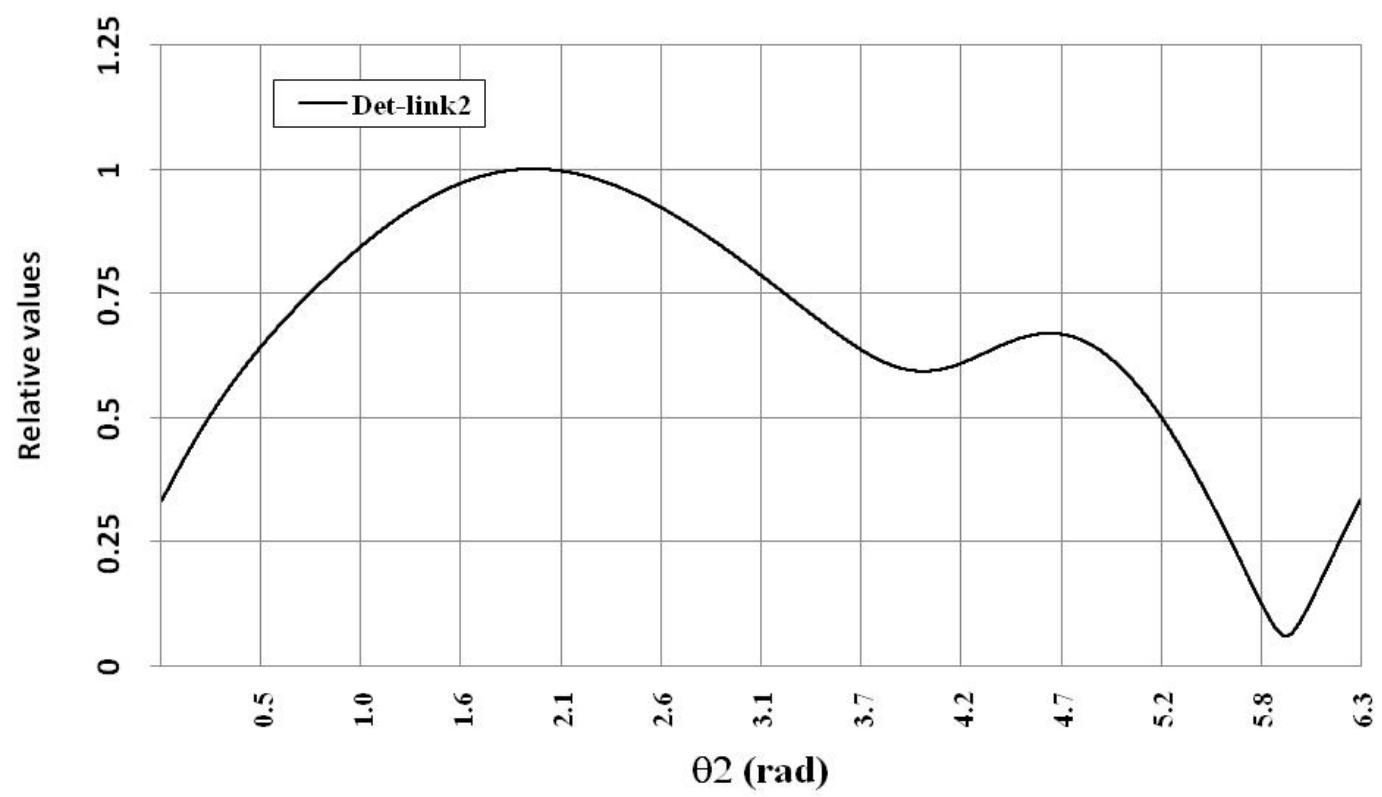

Figure 7. The determinant $\left|\mathbf{J}_{\theta_{2}}\right|$ of the input link

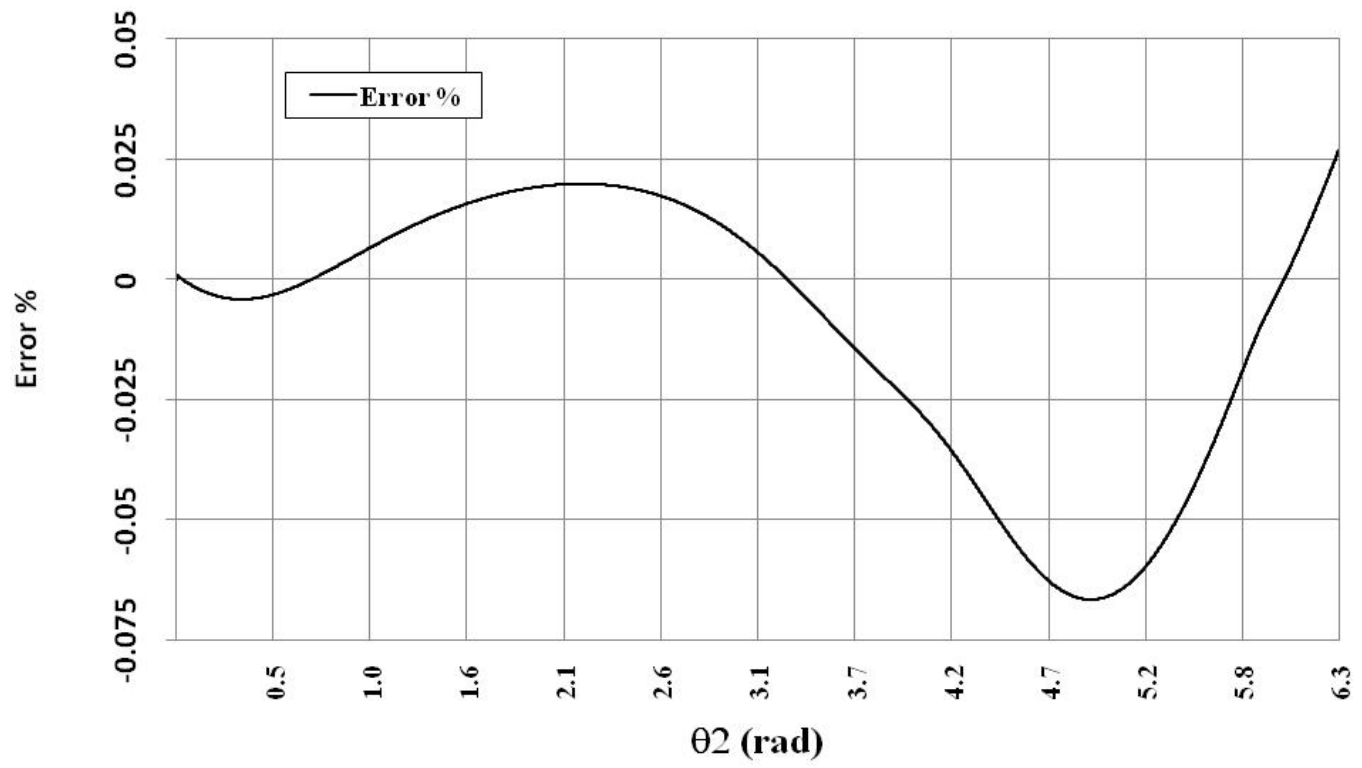

Figure 8. Percentage error of calculations as per equation (14). 
Table 1. Exact and numerical results at sample crank angles.

\begin{tabular}{|c|c|c|c|c|c|c|c|c|}
\hline \multirow{2}{*}{$\theta_{2}(\mathrm{deg}$} & \multicolumn{2}{|c|}{$\theta_{4}(\mathrm{rad})$} & \multicolumn{2}{c|}{$\theta_{5}(\mathrm{rad})$} & \multicolumn{2}{c|}{$x_{6}(\mathrm{~cm})$} & \multicolumn{2}{c|}{$r_{3}(\mathrm{~cm})$} \\
\cline { 2 - 9 } & Exact & Numer. & Exact & Numer. & Exact & Numer. & Exact & Numer. \\
\hline 30 & 0.9994 & 0.999 & 1.5248 & 1.525 & 24.921 & 24.928 & 38.377 & 38.375 \\
\hline 90 & 1.1271 & 1.127 & 2.314 & 2.314 & 6.988 & 7.009 & 47.321 & 47.323 \\
\hline 150 & 1.281 & 1.281 & 2.615 & 2.616 & -4.954 & -4.951 & 41.46 & 41.469 \\
\hline 300 & 1.059 & 1.058 & 1.0517 & 1.054 & 28.414 & 28.444 & 18.861 & 18.817 \\
\hline
\end{tabular}

\section{Conclusions:}

Differential kinematics presents a simplified alternative to closed form analytical expressions needed to study the geometrical behaviour of linkages. For most linkages these closed form expressions are either too messy or not possible to obtain. With computers becoming readily available to students, it is beneficial to introduce differential-model-based numerical methods to these students and encourage them to venture into problems that bear higher levels of difficulty than currently being considered in most academic programs. In this paper, an approach has been presented to facilitate this process. The mathematical procedure is based on the use of kinematic Jacobians matrices, whose determinants offer direct reflections on the mechanism mobility. These matrices have been explained and used in an example that can conveniently be implemented for classroom practice.

\section{References:}

1) Erdman, A. G, Sandor, G. N and Kota, Sridhar., Mechanism Design: Analysis and Synthesis: Vol. 1, Prentice-Hall, New Jersey, 2001

2) Haug, E.J., Computer Aided Kinematics and Dynamics of Mechanical Systems Volume 1: Basic Methods, Allyn and Bacon, Massachusetts, 1989. 
3) Norton, R. L., Design of Machinery, McGraw-Hill, New York, 2001

4) Uicker, J. J., Pennock, G.R and. Shigley, E. J., Machines and Mechanisms, Oxford University Press, USA, 2003

5) Waldron, K.J. and Kinzel, G.L., Kinematics, Dynamics and Design of Machinery, John Wiley and Sons, New York, 1999

6) Ceccarelli, M., “Mechanism Schemes in Teaching: A Historical Overview,” ASME J. Mech. Des. 120, pp. 533-541, 1998 


\section{Appendix}

\section{A closed-form solution for the practice example}

Reference is made to Figure 2, and to points $\mathrm{A}\left(x_{a}, y_{a}\right), \mathrm{B}\left(x_{6}, h_{6}\right), \mathrm{C}\left(x_{c}, y_{c}\right)$ and $\mathrm{O}_{5}\left(x_{05}, y_{05}\right)$ which are shown in the figure. These points are employed to write three geometric constraint equations in three unknowns $\left(x_{6}, x_{c}\right.$ and $\left.y_{c}\right)$ as follows;

$$
\begin{aligned}
& \frac{h_{6}-y_{a}}{x_{6}-x_{a}}=\frac{h_{6}-y_{c}}{x_{6}-x_{c}} \\
& \left(x_{c}-x_{6}\right)^{2}+\left(y_{c}-h_{6}\right)^{2}-r_{4}^{2}=0
\end{aligned}
$$

and

$$
\left(x_{c}-x_{o 5}\right)^{2}+\left(y_{c}-y_{o 5}\right)^{2}-r_{5}^{2}=0
$$

where $x_{a}=r_{2} \cos \left(\theta_{2}\right), y_{a}=r_{2} \sin \left(\theta_{2}\right), x_{o 5}=-r_{1} \cos \left(\theta_{1}\right)$ and $y_{o 5}=-r_{1} \sin \left(\theta_{1}\right)$

It is possible to adopt an iterative Newton-Raphson method to solve the three nonlinear equations for a given $\theta_{2}$ value. However, to obtain a closed-form solution, (ii) and (iii) are manipulated together into the following equation;

$$
\left(x_{o 5}-x_{6}\right)\left(2 x_{c}-x_{o 5}-x_{6}\right)+\left(y_{o 5}-h_{6}\right)\left(2 y_{c}-y_{o 5}-h_{6}\right)+r_{5}^{2}-r_{4}^{2}=0
$$

Also, equation (i) can be used to express $x_{6}$ as follows;

$$
x_{6}=\frac{x_{c}\left(h_{6}-y_{a}\right)-x_{a}\left(h_{6}-y_{c}\right)}{y_{c}-y_{a}}
$$

Now, substitute the expression obtained for $x_{6}$ back in (iv) to develop a $3^{\text {rd }}$ degree polynomial in $x_{c}$ and $y_{c}$ as follows; 


$$
\begin{aligned}
& 2\left(y_{o 5}-h_{6}\right) y_{c}^{3}+\left(r_{5}^{2}-r_{4}^{2}-r_{1}^{2}+h_{6}^{2}+x_{a}^{2}+4 h_{6} y_{a}-4 y_{o 5} y_{a}\right) y_{c}^{2} \\
& +2\left(x_{o 5}-x_{a}\right) y_{c}^{2} x_{c}-2\left(h_{6} r_{2}^{2}+y_{a}\left(h_{6}^{2}-r_{5}^{2}-r_{4}^{2}-r_{1}^{2}-y_{o 5} y_{a}\right)\right) y_{c} \\
& -2\left(h_{6}-y_{a}\right) y_{c} x_{c}^{2}+4\left(h_{6} x_{a}-x_{o 5} y_{a}\right) y_{c} x_{c}+\left(h_{6}^{2}-y_{a}^{2}\right) x_{c}^{2} \\
& -2\left(h_{6}^{2} x_{a}-x_{o 5} y_{a}^{2}\right) x_{c}+\left(r_{5}^{2}-r_{4}^{2}-r_{1}^{2}\right) y_{a}^{2}+h_{6} r_{2}^{2}=0
\end{aligned}
$$

The coordinates, $x_{c}$ and $y_{c}$ can be expressed, as functions of a single variable $t$, as follows;

$$
\left.\begin{array}{l}
x_{c}=x_{o 5}+r_{5} \frac{1-t^{2}}{1+t^{2}} \\
y_{c}=y_{o 5}+r_{5} \frac{2 t}{1+t^{2}}
\end{array}\right\}
$$

$$
\text { where } t=\tan \left(\frac{\theta_{5}}{2}\right)
$$

Substituting (vii) in (vi) will produce a $6^{\text {th }}$ degree polynomial in $t$ as follows;

$$
\sigma_{6} t^{6}+\sigma_{5} t^{5}+\sigma_{4} t^{4}+\sigma_{3} t^{3}+\sigma_{2} t^{2}+\sigma_{1} t+\sigma_{0}=0
$$

where the coefficients $\sigma_{i}$ feature lengthy mathematical expressions constructed of the mechanism's constant dimensions along with $\theta_{2}$. Out of the six roots obtained for this polynomial, only the two real ones will indicate possible solutions. The designer should always select the root which agrees with the initial configuration of the mechanism being studied. Once a root has been selected, it should be inserted in (vii) to find the values of $x_{c}$ and $y_{c}$ which will be subsequently plugged in (v) to find $x_{6}$, and used in $\theta_{5}=\operatorname{atan} 2\left(y_{c}-y_{o 5}, x_{c}-x_{o 5}\right)$ to obtain $\theta_{5}$. The coordinates, $x_{c}$ and $y_{c}$, can also be used to obtain the value of $\theta_{4}$ via $\theta_{4}=\operatorname{atan} 2\left(y_{a}-y_{c}, x_{a}-x_{c}\right)$. Finally, the value of $r_{3}$ is calculated by applying $r_{3}=\sqrt{\left(x_{a}-x_{c}\right)^{2}+\left(y_{a}-y_{c}\right)^{2}}$. This procedure has to be repeated at all the desired values of $\theta_{2}$.

END OF PAPER 


\section{Figure Captions}

Figure 1. A four-bar chain

Figure 2. A linkage used for the practice example - not to scale.

Figure 3. Position, velocity, acceleration and Jacobian determinant of link 4.

Figure 4. Position, velocity, acceleration and Jacobian determinant of link 5.

Figure 5. Position, velocity, acceleration and Jacobian determinant of slider 6

Figure 6. Axial position, velocity, acceleration and Jacobian determinant of slider 3

Figure 7. The determinant $\left|\mathbf{J}_{\theta_{2}}\right|$ of the input link

Figure 8. Percentage error of calculations as per equation (14).

\section{Table Caption}

Table 1. Exact and numerical results at sample crank angles. 